them occupied places where it was impossible they should have been accidental inoculations; once in a most unusual position, the very top of the head in a child with little hair. Since the Esneaux stock was lost, I have never seen a supernumerary vesicle, while the chances of accidental inoculation have remained the same.

A great deal has been said about the uncertainty of animal lymph. I acknowledge there is some truth in this allegation; but I never found this very troublesome. Care goes a long way to rectify it. I believe that this objection would be of no consequence with a good stock, with skill and judgment in cultivation, and with the limitations of the method I have already advocated. I found that, with the lymph from some animals and the avoidance of hurry in its transference, it was equally as certain as human lymph. That this obtains with some calves, seems to carry the inference that it is a matter of judgment in selection. - Of course, in any case, I assume that only the finest vesicles are used.

In revaccination, my success was good ; by counting in every mere approach to a vesicle, it exceeded the percentage obtained in Belgium, namely, 63.2 ; but these of mine were always done direct. Two or three times, I saw troublesome ulcerations follow on each occasion in pale-looking patients, who fainted from the operation. In all cases that succeeded, the febrile symptoms were well marked; in many, rather severely; in several, so much so as to confine them to bed, the measly rash appearing and the axillary glands being much swollen. With these few exceptions, I was quite satisfied with the use of this virus for revaccination.

Through all my experience of animal vaccination in primary cases, I have not once had cause for regret; at first, several gentlemen to whom I supplied it, and who used it with the same amount of insertion to which they had been accustomed with old lymph, were rather alarmed with its severity, and no wonder. In this connection, I should like to refer to a letter of Mr. John Pear: on, Surgeon to the Lock Hospital, published in the appendix to Willan's Vaccine Inoculation, 1806. He says: "When the inoculator makes several parallel and transverse incisions in the same place, the inoculated part inflames violently at the period of efflorescence, and the inflammation often extends from the shoulder to the elbow. The child becomes extremely ill, large erythematous appearances take place on the arm, forearm, the neck, the side, and even on the lower extremity; a fortnight or three weeks will sometimes elapse before the child can be considered free from all danger." He further somewhat superfluously characterises this as an improper vaccination. I have seen this precisely as Mr. Pearson describes from using the new lymph like the old. What is more common now-a-days than this method of insertion which he condemns? The virus of the first human remove from the calf, singularly enough, produces more intense local effect than that from the animal, but this is lost in the subsequent removes. On one occasion, a very remarkable thing happened in the practice of a neighbouring surgeon, to whom I had supplied some of this Esneaux stock. He vaccinated a clergyman's child, six or seven months old, with lymph from the first human remove ; the vaccinifer was healthy. I was desired to see the case on the twelfth day, and found a very much inflamed and swollen arm, with a series of from twenty to thirty full-sized vesicles exactly like small-pox, grouped irregularly upon the upper arm, lying thickest about the seat of insertion. No other vesicles appeared on any other part, and the case did well ultimately.

Of the lymph-stock of Vauves, which in I870 superseded that of Esneaux, I have little to say. It was at first pretty much the same as the latter, but after a time it ceased to possess those active qualities that have here drawn my praise. I became dissatisfied with it; it no longer had the brilliancy and power of the Esneaux lymph, nor its infective qualities. The particular advantages to which I have referred, as manifested by the former alike on the calf as on the human subject, were categorically absent in degree. I was of opinion that it no longer accomplished the great raison d'être of animal vaccinationnamely, the supply of a lymph of as high a standard relatively to variola as judiciousness and safety would permit. The grounds for my dissatisfaction I found to exist alike, whether I cultivated the virus from calf to calf, as I had hitherto successfully done, or inoculated the animals each time with a fresh supply from Belgium. I therefore, about fourteen months ago, discontinued to reproduce the lymph on my own calves; but I have always held that the advantage which calfvaccination gives to overcome the strong dread in some people of the transmission of diathetic diseases by human lymph, was worthy of attention in a subsidiary or collateral way. For this reason, I still continued to procure and use Belgian lymph when it was requested, and because, although it would not bear comparison with the Esneaux virus, it did give a fair result, equalling good human stocks, minus, however, their certainty. The present lymph in use in Belgium has been derived from Milan. My knowledge of it is limited to its action on the human subject, and from tubes alone. It would be unfair, therefore, to base any strict comparison upon this, but I am fully able to state that I am satisfied with the course and appearance of the vesicles raised from it, and with the signs of constitutional action; but, no matter how good the present stock may be, it does not affect the argument of my paper, nor is it possible that this should be neutralised by any matter-of-fact explanation.

Now, having arrived at this point, let me, in the briefest possible way, consider what is the prospect of finding a remedy for this precarious nature of the virus. I appeal to the Government to institute systematic efforts to discover fresh cases of cow-pox, and, when found, to take the lymph and experiment for themselves. Are they not morally bound to seek the truth by investigations that shall take cognisance of all well-advanced assertion; and, if it should be found that animal vaccination is either pernicious per se, or likely to become so by mismanagement to a greater degree than by mismanagement of merely human vaccination, which I affirm is true, then, is it not imperative that the practice of the new method in private hands should be forbidden by enactment? On the contrary, if it be found advantageous, must they not adopt it, to some extent, as one of their resources, on the same principle as that on which the Vaccination Act itself stands?

It is with very great pleasure that I hear the authorities have determined to repeat the experiments of that distinguished member of the Association, Mr. Robert Ceely, upon the artificial production of cowpox, for it is this that should form the real basis of effort in the direction of new lymph-supply. Naturally, a fresh case of cow-pox could not always be forthcoming just when it was wanted.

I am one of the few who have been successful in producing artificial cow-pox; and, though I have tried many times, have not again succeeded. It is tempting to enter upon this question fully, but time will not allow. I hear that the first set of experiments conducted by the authorities have failed; no doubt; they will try again till they do succeed. I should look hopefully to increased experience to remove the difficulties attending this operation. Were I again engaged in this attempt, I should select new milch-cows with their first or second calf, in the very highest condition of health possible, with their skins full of the juices of the rich summer grass. I should select amongst the number several white cows and Alderneys. I would do the inoculation by deep incision with variolous lymph of not too late a stage, and from a sample mixed from several cases. I should use it with and without glycerine; the air of the stables I would have rather warm and very moist. I think premature desiccation is one of the difficulties.

In the case in which I was successful, I did not wait till the vesicle had quite formed, but took matter from the most prominent of the tubercles, passed it consecutively through two calves, and then on to two children, obtaining in one of them two magnificent cow-pox vesicles, and nothing more.

Gentlemen, we are in the presence of a crisis in the history of vaccination. Nations, dissatisfied with the spread of small-pox, yet still trusting in the kindly prophylactic, turn their eyes towards the new manner of direct inoculation. Now, more than ever, must our noble profession guide our country wisely. This favoured land, that first received the fairy gift through the wisdom of Jenner, must not lose it through the folly of his successors. We must refuse to adopt this Neapolitan practice to the extent advocated ; it is cumbrous, precarious, and far more liable to mismanagement than is our present system. Let us rather take a lesson from the practical botanist, who, shaking fresh pollen into his old bloom, produces a more vigorous specimen. Let us find means, through the calf, to renew our old stocks at will from the "variolæ vaccinæ". That lymph which gives the greatest constitutional affection of the right kind for the smallest number of insertions will give the greatest local beauty, and well-nigh absolute security from variola.

\section{ERYSIPELAS AND VACCINATION.*}

\section{By SAMUEL LODGE, M.R.C.S., Bowling, Bradford.}

IT is with some diffidence that one approaches the subject of erysipelas here, in this hospital and in the presence of such a number of distinguished practitioners ; nevertheless it may be that the disease, considered as a result of septicæmia in connection with vaccination, and treated by what I have al'ways designated the Leeds method, could not be more appropriately discussed by any society than by the West Riding Medico-Chirurgical. Mentioning to my friend Mr. F. Hall

\footnotetext{
* Read before the West Riding Medico-Chirurgical Society.
} 
last year-who had spoken to me of one or two cases of erysipelas following vaccination in Leeds-that I always used the tincture of perchloride of iron, I think he replied that it had been tried in the cases alluded to without effect. At the first glance, this answer looked discouraging enough; but I question if further observation and experiment do not enable us, as vaccinators, to look upon the disorder with confidence and serenity, rather than with apprehension and dread. I have seen two cases of fatal erysipelas in children, and the treatment by iron was resorted to in both. I make no difficulty in admitting that, whatever had been done for those unfortunate creatures by way of remedies taken or used, the result would have been the same. The cellular parts of the bodies seemed disorganised precisely as we have all observed them in deep burns-the connecting tissues rotted away; but in neither of these cases was anything suspected as traceable to vaccination. They were purely idiopathic; they occurred at different periods, in different places, and did not appear at all contagious.

It is now proper to notice one particular direction of the vaccination authorities. We are "not to use virus from vesicles surrounded by inflamed areolæ". Why? Because the inflammation may be erysipelas, probably. There seems to be some mistake here. Observation has taught me that the inflamed areola, as ordinarily seen, is not erysipelas; and that, if it be only brushed over with a weak solution of iron, it will certainly subside without further trouble. Of course, it is not to be inferred that the red ring now spoken of will end in erysipelas if let alone. On the contrary, the contention is, that this distinguishing mark of keen virus and active circulation will, in most cases, return to the natural condition as the vaccine disorder exhausts itself. But, to make the matter perfectly safe, it is only necessary, after puncturing the vesicles, to treat the inflammation in the manner above described. A step further takes us into a more doubtful and dangerous region. If, after vaccination, beyond the inflamed surface - say near the elbow-joint, but divided from the vesicular inflammation by a circular line of raised hard pale skin - there should appear a patch of dull red colour, that is quite another matter, and I should not recommend that lymph from such an arm should be used.

The erysipelatous circles or spots, or whatever shaped discoloration the skin may assume, must be treated with the iron at once; and wherever the diseased patches may extend, to them must its application be continued.

I have seen erysipelas as the result of vaccination in three cases, and in one of them almost every part was attacked and painted over. The three cases referred to occurred in our own practice about four years ago. There was nothing uncommon about the appearance of the vesicles; in two of them, the cow-pox inflammation was but slight. I did not see the third until afterwards. Finally, they all recovered. From that time, I have always applied the tincture of perchloride of iron to any suspicious-looking arm; and since having found every painted case to do well, I now use it in all without distinction. In fact, mothers and nurses would not contentedly take their charges away if the treatment were omitted.

Mention has already been made of one particular direction by the vaccination authorities to public vaccinators, and we now proceed with due humility to remark upon another.

It is somewhat unquestionable how far the Hunterian dogma is correct, that two disorders having similar characteristics cannot exist at the same time in one person; but it is difficult to divest one's mind of long-conceived impressions, and perhaps the following case may be interesting to some practitioners who, like myself, do not entertain the belief of all that is old being false and all that is new true. Small-pox was raging in a part of our district, and we were engaged in revaccinating the people. The grown-up daughter of a man who was lying ill of the disease came to be vaccinated. She had not been vaccinated in early life. I made the usual light scarifications, scarcely drawing blood, when the woman, without any apparent suffering, suddenly fell down in a swoon. It was thought that a fine strong young woman should not faint from so slight a cause; and, when she recovered, such answers were obtained as convinced us of her having been ill before coming to the station. This actually proved to be the case, for in a few days a regular crop of small-pox pustules appeared. The disorder ran through its stages, and the woman recovered well. I need hardly say that I anxiously watched for the advent of the cow-pox; but it never came.

It is possible that two analogous diseases may exist in the same person at the same time; but it does not seem easy to believe it. Then what are we to think of this direction to public vaccinators: "You are to be careful not to convey the blood of the patient you are operating upon back to the vesicle of the child from whose arm you are taking the lymph"? Water, a cloth, etc., are always to be used--and by me always are used-to purify the lancet after every time it touches the new patient, in order that the blood of a child suffering from cow-pox at its greatest height may not be contaminated. Now, bearing in mind that the surface to which the lancet is applied for lymph is not an absorbing, but a rapidly exuding one, I venture to suggest that the government theory is physiologically incorrect. Right or wrong, however, this direction cannot do harm; and at any rate it necessitates caution.

My friend Mr. Parkinson of Bradford assures me that he now vaccinates with the same lancet that he has used for thirty years, and that he has never met with a case of disease consequent upon vaccination performed by himself or anybody else. Dr. Foster of Leeds, who has been a vaccinator over forty years, declares that he, in all his experience, has never seen a bad result from vaccination, nor even any approach to such a thing.

I have sometimes operated, according to the instructions of Dr. Stephens, by a number of small scarifications; at other times, by the tattoo dots of Dr. Willshire; and very often by the long line of the oldest practitioners; and, I am bound to say, with a like good result in all, so far as the prevention of small-pox has been concerned. In short, I may say that I have never known that terrible disorder occur to a patient that I have effectively vaccinated.

Fearing that I may be misunderstood, let me add that I have treated small-pox through three epidemics; that I have known pregnant women to suffer from it, the lives of both themselves and their offspring being sacrificed; and, finally, that I have attended one patient who died of a second confluent attack.

\section{ABSTRACT OF A PAPER ON VACCINATION AND REVACCINATION.*}

By F. P. ATKINSON, M.D., Kingston-on-Thames.

DR. ATKINSON said it was now generally acknowledged-

I. That vaccination lessens the chances of taking small-pox, though it does not render the reception of it in after-years altogether impossible.

2. That vaccination, in most cases, greatly modifies the character of the eruption and lessens the severity of the attack.

3. That revaccination gives an almost absolute immunity from smallpox, provided it is efficiently performed after fifteen years of age.

The following evidence was then produced in proof of the correctness of the views held.

In the Punjaub, with a population of $18,000,000$, the deaths from small-pox, according to the statement of Dr. A. C. C. De Renzy, sanitary commissioner in the province, are never less than 20,000 a year. In 1869, they numbered 53,195. In England, the average annual mortality does not exceed five thousand, though, previously to the introduction of vaccination, it was quite as high as in the Punjaub. Dr. Brett, Medical Officer of the Watford Rural Sanitary Authority, states that, in 1877 , small-pox visited a family, nine in number, residing at Bushey; three had been vaccinated, six had not. All the six unvaccinated persons took the disease, and four died; while the three that had been vaccinated escaped altogether, one of the three beingr a child at the breast, fed by its mother within two hours of her death.

Dr. Schwarzgruber of Harland, in the Medicinisches Chirurgisches Centralblatt, 1877, shows the marked efficacy of vaccination in the epidemic of small-pox which appeared in August of that year. The first patient was a girl aged 5 , who had not been vaccinated. She had the disease in a severe form, and, after ten days' illness, died. In the same house was another family with four children ; two vaccinated and two unvaccinated. The two unvaccinated-a girl aged is and a boy aged $21 / 2$-took the disease and died; the two vaccinated children escaped. A third family was visited by the disease; in it also were four children-two vaccinated and two unvaccinated. The two latter had small-pox, and one died; while the two vaccinated escaped, though constantly exposed to the infection. In a fourth family, an unvaccinated girl aged 9 had a dangerously severe attack of small-pox, but escaped with the loss of an eye. A vaccinated boy aged 4 , in the same family, had the disease in a very mild form. In a fifth family of eight children, of whom seven were vaccinated, six of the vaccinated had mild attacks of small-pox (four not being confined to bed); while, in the unvaccinated child, the disease proved fatal.

The course of events in other families was similar. In all, there were thirty cases of small-pox among vaccinated persons, with only one death, which may be attributed to the patient having gone, while scantily clothed, from a warm room into the cold air. Of the non- 